Artículo de investigación

Cómo citar: Hernández, G. (2020).

La dialéctica argumental de

Hubert Marraud en la enseñanza

universitaria de la argumentación.

Polisemia, 16 (30), 83-102.

http://doi.org/10.26620/uniminuto.

polisemia.16.30.2020. 83-102

ISSN: 1900-4648

elSSN: 2590-8189

Editorial: Corporación Universitaria Minuto de Dios - UNIMINUTO

Recibido: 5 de Julio 2020

Aceptado: 15 de agosto 2020

Publicado: 15 de octubre 2020

\title{
La dialéctica argumental de Hubert Marraud en la enseñanza universitaria de la argumentación
}

\author{
Hubert Marraud's Arguments' \\ Dialectics in University Teaching \\ of Argumentation
}

\author{
A dialéctica de argumentação \\ de Hubert Marraud no ensino \\ universitário da argumentação
}

\section{Resumen}

Hubert Marraud ha desarrollado una propuesta de análisis y evaluación de argumentos, desde la perspectiva lógica en teoría de la argumentación, a la que llama dialéctica argumental, según la cual el carácter dialéctico en argumentación no se presenta solo entre los argumentadores, sino también entre los propios argumentos internos de los discursos argumentativos. A partir de su propuesta ha ofrecido, desde hace casi diez años, un curso de argumentación a estudiantes de Filosofía en la Universidad Autónoma de

Gabriela Hernández Deciderio

Escuela Nacional Preparatoria, Universidad Nacional Autónoma de México.

Correo electrónico: gabriela.hernandez@enp.unam.mx

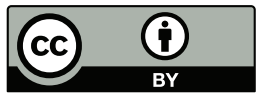

Madrid. En este escrito se presenta la propuesta dialéctica argumental, la integración de temas y la metodología con las que Marraud confecciona su curso universitario, así como reflexiones sobre algunos de los resultados alcanzados. Esta exposición pretende evidenciar el singular beneficio recíproco que se logra al combinar investigación y enseñanza en teoría de la argumentación.

Palabras clave: teoría de la argumentación, perspectiva lógica en argumentación, dialéctica argumental, análisis de argumentos, evaluación de argumentos. 


\begin{abstract}
Hubert Marraud has developed a proposal for the analysis and evaluation of arguments from the logical perspective in argumentation theory, which he calls arguments' dialectics, according to which the dialectical character in argumentation does not appear only among the arguers, but also between the arguments themselves within the argumentative discourses. Based on his proposal Marraud has offered, for almost ten years, an argumentation course to Philosophy students at the Universidad Autónoma de Madrid. In this paper, I offer a presentation of the arguments' dialectics proposal, the integration of themes and the methodology Marraud presents in his college course, as well as considerations on some of the results achieved. This exhibition intends to show the uniqueness of the mutual benefit achieved by combining research and teaching in argumentation theory.
\end{abstract}

Keywords : theory of argumentation, logical perspective in argumentation, argument dialectics, analysis of arguments, evaluation of arguments.

\title{
Resumo
}

Hubert Marraud desenvolveu uma proposta para a análise e avaliação de argumentos, da perspectiva lógica da teoria da argumentação, a que chama dialéctica argumentativa, segundo a qual o carácter dialéctico na argumentação não está presente apenas entre os argumentistas, mas também entre os argumentos internos dos discursos argumentativos. Com base na sua proposta, ofereceu, durante quase dez anos, um curso de argumentação a estudantes de Filosofia na Universidad Autónoma de Madrid. Nestedocumento apresentamos a proposta de argumentação dialéctica, a integração de temas e a metodologia com que Marraud desenvolveu o seu curso universitário, bem como reflexóes sobre alguns dos resultados alcançados. Esta apresentação visa demonstrar o benefício recíproco único que é alcançado ao combinar a investigação e o ensino na teoria da argumentação.

Palavras-chave: teoria da argumentação, perspectiva lógica da argumentação, dialéctica da argumentação, análise da argumentação, avaliação da argumentação. 


\section{Introducción}

Hubert Marraud no solo es un estudioso de distintas líneas de investigación en la teoría de argumentación, sino que, desde la perspectiva lógica de la argumentación, ha desarrollado una propuesta teórica a la que llama dialéctica argumental o dialéctica de los argumentos. En ella plantea que, además de la interacción dialéctica entre los participantes de una situación argumentativa, también es posible realizar un análisis dialéctico de los argumentos que ofrecen los argumentadores, ya que es posible captar el macroargumento que construyen interactivamente y que queda plasmado en un texto o un discurso.

A lo largo de casi una década de impartir un curso de argumentación en la Universidad Autónoma de Madrid (UAM), el trabajo de Marraud ha dejado constancia de que las necesidades didácticas requeridas en la enseñanza universitaria estimulan el desarrollo de la investigación en teoría de argumentación; y recíprocamente, sus progresos en la investigación fortalecen su práctica didáctica. Ese mutuo beneficio que se presenta al vincular investigación y enseñanza también se aprecia en la obra escrita de Marraud, ya que en ella da cuenta de la manera en que, durante la última década, ha ido afinando tanto su propuesta teórica como su modelo de enseñanza.

En este artículo, explicaré en qué consiste la propuesta de la dialéctica argumental, para después apreciar cómo es que se integra en el diseño de un curso universitario de argumentación. Revisaré con detenimiento el diseño temático y didáctico del curso y para exponerlo con mayor precisión, detallaré las competencias que detecté se persiguen en cada uno de los tres momentos en los que Marraud organiza la exposición de su curso. Destacaré las características de la enseñanza de la argumentación en el modelo universitario de la Facultad de Filosofía de la UAM, pues reconocerlas es una guía para apreciar la coherencia existente entre la orientación del curso (en su sentido teórico y práctico) y las decisiones didácticas que se adoptan para su desarrollo, así como en la selección de los materiales de respaldo o en el diseño de los ejercicios y de los instrumentos de evaluación. Asimismo, para tener una idea más amplia del curso y de sus frutos, considero oportuno ofrecer un pequeño análisis estadístico de los valores alcanzados por los alumnos en las tres test que son el principal instrumento de evaluación del curso. ${ }^{1}$ Analizo los puntajes obtenidos por los alumnos de los dos grupos cursaron la asignatura, en los cursos de 2017 a 2019, que en total conformaron un universo de 205 alumnos. Presentaré únicamente los resultados globales, con la intención de ofrecer algunos datos duros, que si bien son insuficientes para tener una idea detallada de los resultados del curso, al menos proporcionan indicios de la respuesta que están mostrando los estudiantes ante éste. El análisis de esos datos pondrá de manifiesto que para tener una idea más clara y detallada de los resultados del curso hace falta algo más que una mera revisión cuantitativa.

1 Agradezco ampliamente a Hubert Marraud todas las facilidades que me brindó para asistir a las clases de su curso de 2019 y, sobre todo, la confianza con la que me proporcionó los resultados numéricos obtenidos por sus estudiantes en los cursos de 2017 a 2019. 
Una vez que conozcamos en qué consiste la propuesta de dialéctica argumental, cómo se integra en el diseño de un curso universitario y algunos indicios de los resultados que está teniendo en los estudiantes, mostraré elementos que nos ayuden a apreciar cómo se ha dado un beneficio recíproco entre investigación y enseñanza en el trabajo de Hubert Marraud. Finalmente exploraré alguna razón que explique cómo es que ese mutuo beneficio, entre investigación y enseñanza, se presenta de un modo singular en el campo de la teoría de la argumentación.

Así, el desarrollo de este estudio consta de cinco apartados. En el primero, presentaré la dialéctica argumental, explicando cómo es que corresponde a la perspectiva lógica ${ }^{2}$ en teoría de la argumentación y no, pese a su nombre, a la perspectiva dialéctica. En el segundo apartado expondré el diseño y didáctica del curso universitario que toma las bases del modelo dialéctico argumental. En el tercer apartado ofreceré un pequeño análisis estadístico de los resultados de los test aplicados durante el curso en los últimos tres años, como medio para tener algún indicio de la respuesta de los estudiantes al curso. En el cuarto apartado examinaré con más detalle cómo se presenta y qué características tiene el mutuo beneficio que se da entre investigación y enseñanza en el trabajo de Marraud, así como una reflexión sobre si ese mutuo beneficio se presenta de manera singular en el campo de la teoría de la argumentación. En el quinto y último apartado, ofreceré algunas conclusiones.

\section{La propuesta dialéctica argumental como parte de la perspectiva lógica en la teoría de la argumentación}

El estudio de las prácticas argumentativas es atractivo para distintos teóricos, entre los que destacan lingüistas, filósofos y teóricos de la comunicación; los diversos intereses de investigación que han desarrollado pueden comprenderse desde distintas perspectivas. Uno de los autores que mejor ha expuesto esta visión perspectivista en argumentación es Joseph W. Wenzel, que en su ensayo Perspectives on Argument (1992, pp. 136-140) plantea cuatro razones por las cuales es útil reconocer las distintas perspectivas en los estudios en argumentación. De manera resumida, las razones son las siguientes:

1. Ayudan a clarificar el significado de trabajos anteriores y futuros sobre la argumentación y sus líneas de investigación.

2. Pueden servir para clarificar diferentes clases de estudios críticos o evaluativos, así como para identificar «seudoproblemas». ${ }^{3}$

2 Tomando el término "lógica" en un sentido amplio, como teoría de los argumentos.

3 Wenzel (1992, pp. 137-138) plantea por ejemplo el debate sobre la relevancia de la lógica con respecto a la retórica en el que se involucraron Mortensen y Anderson, por una parte, y Mills y Petrie, por otra. 
3. Sirven para exigir un tratamiento más simple y completo del campo de la argumentación.

4. Ayudan a reconocer las distintas perspectivas en una base de igualdad, por el hecho de ser una de entre varias perspectivas.

Wenzel realizó un primer estudio en 1990, titulado Three Perspectives on Argument, en el que explica que la retórica, la dialéctica y la lógica no son tres tipos de argumentación, sino tres maneras de estudiarla. Esas tres perspectivas son, según este autor, diferentes formas de entender cualquier caso de comunicación humana que parece involucrar argumentos, puesto que revelan diferentes aspectos de cualquier instancia de la argumentación, al modo como en un edificio se pueden apreciar sus tres planos: frontal, lateral y superior (Wenzel, 1990/2006, p. 9). Aunque estas tres perspectivas no son las únicas que se han propuesto en la literatura especializada, ${ }^{4}$ por cuanto sabemos de ellas desde los estudios antiguos, podemos reconocerlas como las perspectivas clásicas.

La mayor virtud de la caracterización que nos ofrece Wenzel en sus estudios sobre las perspectivas clásicas es que reconoce con nitidez la diferencia en objetos de estudio de cada una: identifica que la perspectiva retórica se centra en el «argumentar» visto como un «proceso», la perspectiva dialéctica se centra en la «argumentación» como un «procedimiento», y la perspectiva lógica se centra en los «argumentos» como «productos» (Wenzel, 1992, p. 134). Deja en claro que cada una de las perspectivas surge de motivaciones distintas. La perspectiva retórica, en sentido práctico, se pregunta por las formas de alcanzar la persuasión y, en sentido teórico, por comprender las condiciones de la argumentación efectiva. La perspectiva dialéctica, en sentido práctico, se ocupa de ejercer la crítica y, en su sentido teórico, busca la forma de reglamentar procedimientos argumentativos para que sean transparentes. Por último, la perspectiva lógica tiene el sentido práctico de emitir juicios sobre los argumentos como productos de la argumentación y el propósito teórico de establecer estándares de argumentación correcta. Pero Wenzel (1992, p. 123) enfatiza que, al hablar de perspectivas en teoría de la argumentación, hablamos de propuestas teóricas desde las que se analizan y entienden las prácticas argumentativas y no de los fenómenos argumentativos perse.

La propuesta teórica dialéctica argumental de Hubert Marraud plantea que es necesario analizar las relaciones entre argumentos de un mismo discurso. Y aunque reconoce una dialéctica ${ }^{5}$ entre los argumentos, no por ello ubica su propuesta en una perspectiva dialéctica de los estudios en

4 En distintas publicaciones, además de reconocer las tres perspectivas de las que habla Wenzel, se propone alguna más. Así, por ejemplo, Luis Vega $(2017$, p. 5) nos habla de una perspectiva socio-institucional, Lilian Bermejo-Luque $(2018$, p. 10) reconoce una perspectiva epistemológica y Hubert Marraud (2019, pp. 13-14) nos habla de una perspectiva lingüística.

5 A lo largo de la historia, la denominación dialéctica hace referencia a la idea de oposición; por eso Hubert Marraud la recupera para hablar de oposición entre argumentos. 
argumentación, sino más bien dentro de una perspectiva lógica, puesto que continúa centrándose en los argumentos vistos como productos. Pero no como productos individuales, sino en la relación entre argumentos en el mismo juego discursivo. Identifica las relaciones existentes entre los argumentos, relaciones que son detectadas mediante el análisis de ciertas partículas lingüísticas (como: además, pero, por otra parte, etc.) presentes en los argumentos. Marraud observa que esas partículas expresan relaciones de oposición, refuerzo o comparación. Y si son vistas como relaciones de oposición, no lo son en el sentido de la oposición del cuadro tradicional de oposiciones, sino en un sentido mucho más cercano a la oposición de la dialéctica filosófica, entendida como la relación entre tesis, antítesis y síntesis. El objetivo de la dialéctica argumental es desarrollar criterios para comparar y evaluar la fuerza relativa de los argumentos, así como clasificar los contraargumentos tomando en cuenta el efecto que se busca con su uso.

Entonces, la propuesta dialéctica argumental permite establecer una diferencia entre dos maneras de entender la dialéctica, que Hubert Marraud (2017b) explica de la siguiente forma:

Hay dos concepciones básicas de la dialéctica. Puede referirse al arte de la controversia o del debate, con confrontación de opiniones y de argumentadores. La dialéctica así entendida se centra en las reglas y procedimientos convencionales que rigen esas confrontaciones. Es lo que he llamado (Marraud, 2015) «dialéctica argumentativa». La dialéctica también puede referirse al estudio de las oposiciones entre argumentos. Esta concepción presupone un concepto comparativo de argumento convincente, y puede definirse como el estudio de la fuerza de los argumentos. El propósito de esta «dialéctica argumental» es desarrollar estándares y criterios para comparar y evaluar la fuerza relativa de los argumentos. La propuesta de una clasificación de los contraargumentos basada en el efecto buscado con su uso pretende ser, pues, una contribución a la dialéctica argumental. (p. 2)

La dialéctica argumental corresponde a la perspectiva lógica porque se centra en los argumentos como productos de las prácticas argumentativas y en las relaciones entre ellos. Y no se interesa por las reglas que regulan lo que está o no está permitido a los argumentadores en los procedimientos argumentativos, que es lo que estudia la perspectiva dialéctica $y$, en los términos de Marraud, corresponde a una dialéctica argumentativa y no a una argumental.

Pero la dialéctica argumental, además de mostrar dos formas de entender la dialéctica, también permite reconocer dos maneras de desarrollar la perspectiva lógica, porque muestra dos maneras de realizar un análisis lógico de los argumentos en el campo de la teoría de la argumentación. Hay un análisis lógico en teoría de argumentación que se dedica únicamente a estudiar la estructura interna de los argumentos; es decir, se enfoca en el análisis de las relaciones inferenciales: el se sigue que, que se da entre premisas y conclusión en el interior de los argumentos individuales. A ese tipo de análisis se han dedicado especialmente teóricos estadounidenses y 
canadienses, en buena medida identificados con el movimiento de lógica informal. ${ }^{6}$ En cambio, la dialéctica argumental muestra que además es posible estudiar relaciones entre argumentos, como la relación es más fuerte que, la cual se presenta entre los argumentos de un mismo proceso discursivo.

El desarrollo de la propuesta dialéctica argumental se dirige con naturalidad al contexto de su enseñanza, puesto que los criterios para comparar y evaluar la fuerza de los argumentos o para comprender la contraargumentación, han de ser enseñables. Así que el paso de la teoría a la enseñanza es espontáneo, pero la consolidación de un curso que logre los fines educativos que se deseen, no es un hecho automático; requiere desarrollar procesos didácticos y metodológicos que además sean coherentes con las necesidades de la institución educativa.

\section{La dialéctica argumental de Hubert Marraud en el diseño de un curso universitario}

El curso Lógica y Argumentación, dictado por Hubert Marraud en la Universidad Autónoma de Madrid (UAM), está dirigido a estudiantes universitarios del primer semestre del grado en Filosofía, pero pueden matricularse estudiantes de otras carreras universitarias, específicamente de los grados en matemáticas o en historia y ciencias de la música. Así, entre el 20 y el $30 \%$ de los alumnos matriculados en la asignatura provienen de carreras diferentes a la de Filosofía. Además, la UAM admite que en sus programas universitarios se matriculen estudiantes de mayor edad que aquellos recién egresados de la educación media, de tal manera que entre un 4 y $8 \%$ de los alumnos matriculados en el curso de Lógica y Argumentación, son de edades mayores. Es importante tomar en cuenta las características del alumnado para definir el objetivo del curso y establecer su orientación; esto es, definir si se encaminará solo a la formación teórica, a la investigación, o si se ofrecerán herramientas prácticas. Esa decisión determinará el tipo de materiales del curso y permitirá ver si los instrumentos de evaluación son coherentes. Hubert Marraud ha determinado una orientación teórico-práctica para su curso y lo imparte a dos grupos por semestre, en un horario matutino (grupo 110) y vespertino (grupo 160).

En la definición del objetivo del curso, Marraud parte del hecho contundente de que este no se dirige a enseñar a argumentar a los estudiantes, puesto que ellos saben hacerlo y lo hacen. El objetivo central es ofrecer herramientas prácticas encaminadas a que los estudiantes desarrollen las competencias que les permitan no solo analizar y evaluar los argumentos

6 Entre sus autores más representativos están Anthony Blair, Ralph Johnson y Trudy Govier.

7 El grupo 110 suele tener entre un 50 y un 60\% más alumnos matriculados que los que asisten a clase en el grupo 160. Las clases son de tres horas semanales, dos para revisar teoría, ejemplos y ejercicios guiados y una más especialmente para realizar ejercicios. Para acudir a la hora exclusiva para ejercicios, cuando el grupo supera los 20 estudiantes matriculados, se divide en dos. 
propios o propuestos por otros, sino también comparar la fuerza relativa de los argumentos, tomando en cuenta el efecto que se busca al usarlos, para que así sean capaces de explicar y justificar a alguien más cuándo se argumenta debidamente y cuándo no.

Para lograr ese fin, el curso se desarrolla en tres momentos, y al término de cada uno, el alumno ha de presentar un test, que contiene ejercicios similares a los que fue realizando durante las clases, para medir su desenvolvimiento en las competencias consideradas en ese periodo. Para obtener una visión global del curso y apreciar la forma en la que el modelo «dialéctico argumental» da sentido al objetivo central, conviene revisar brevemente el contenido general que se ofrece en cada uno de los tres momentos del curso:

En el primer momento del curso, se plantean los conceptos generales que sirven para describir las prácticas argumentativas, puesto que sirven para identificar los elementos que integran a los argumentos y para diferenciarlos de otros productos lingüísticos.

En el segundo momento, se desarrolla una teoría de análisis, que proporciona los conocimientos para poner en práctica la identificación de argumentos simples y de argumentos complejos; en este momento se adquiere el conocimiento sobre tipos de argumentos y operaciones que resultan indispensables para la evaluación.

En el tercer y último momento del curso, se desarrolla una teoría crítica de los argumentos, dirigida a la evaluación de argumentos simples, pero sobre todo a la evaluación de argumentos complejos. Es el momento del curso en el que se hacen evidentes las conexiones interargumentativas en un mismo discurso: el carácter dialéctico de los argumentos.

Así, en el desarrollo del curso, la propuesta dialéctica argumental está presente con claridad desde el segundo momento, pues ofrece las claves para reconocer los tipos de argumentos, el encadenamiento, la coorientación y la metaargumentación, que son elementos indispensables para comprender que entre los argumentos de un mismo proceso discursivo se establecen relaciones de oposición, refuerzo o comparación. De modo que, en el tercer momento del curso, es posible retomar los elementos de la teoría de análisis, pero con un enfoque evaluativo para ejercitarse en la ponderación de argumentos y para comprender la mecánica que opera en la contraargumentación, dependiendo del fin que se persiga. Una de las herramientas más importantes que ofrece el curso, tanto para reconocer estrategias para atacar argumentos como para identificar qué argumento posee mayor fuerza argumentativa, es el uso de esquemas argumentativos y de las preguntas críticas asociadas a cada uno. En la propuesta de Marraud, los esquemas se organizan en tres clasificaciones (generales, representativos y directivos), que dan lugar a veintidós tipos distintos en total. En el desarrollo del curso, cada uno de sus tres momentos presupone el anterior e implica pasar de contenidos y ejercicios simples a otros de mayor complejidad. 
Considero que un recurso pertinente para conocer con detalle las estrategias didácticas y su vinculación con los temas de la asignatura es especificar las competencias que se espera que los estudiantes desarrollen durante cada uno de los tres momentos del desarrollo del curso. Parto del entendido de que una competencia es un comportamiento observable que se activa ante una situación específica que demanda una respuesta. Lograr dar esa respuesta supone haber sido capaz de integrar los saberes, las habilidades requeridas, así como las actitudes apropiadas para ese logro. ${ }^{8}$ Aunque el término competencia tiene todavía una serie de problemas, ${ }^{9}$ debe quedar claro que hablar de competencias no significa referirse simplemente a la unión de conocimientos, habilidades o actitudes, puesto que lo que hace una competencia es movilizar, integrar y orquestar tales recursos. Se trata de una movilización que solo resulta pertinente en situación, y cada situación es única, aunque se la pueda tratar por analogía con otras ya conocidas.

Definir competencias argumentativas, desde la óptica de un curso de argumentación, significa considerar competencias específicas y no simplemente competencias básicas o transversales. ${ }^{10}$ La redacción de una competencia específica incluye la enunciación del verbo de desempeño que indica lo que buscamos hacer y nos ubica en la habilidad guía; luego se especifica el objeto conceptual, que da las bases cognoscitivas para el hacer y que responde a la pregunta por el qué es lo que se sabe para la acción. $\mathrm{Y}$, por último, se plantea la situación de referencia en la que se ejecuta la acción o las acciones específicas, de modo que responda a preguntas por el para qué, cuándo y cómo, al que se orientan las actitudes. Entonces, para la redacción de una competencia específica podemos seguir la secuencia: Verbo de desempeño + objeto conceptual + contexto o situación de referencia para la acción. ${ }^{11}$

Tras asistir a las clases presenciales del curso Lógica y Argumentación (2019-2020) y revisar el programa, así como los materiales e instrumentos

8 Para un panorama más amplio sobre las competencias, véase Trujillo (2014).

9 Según Manso y Sánchez-Tarazaga (2020), el término competencia dio problemas por la forma en la que se le introdujo en el ámbito educativo, pues los distintos sectores involucrados en esa introducción las interpretaron a su manera particular, lo cual dio lugar a la aparición de diferentes y variadas definiciones, generando así confusión y desconcierto. Además, se propuso el concepto sin ofrecer ejemplos de experiencias concretas, lo cual generó imprecisión y la percepción de que tan solo se introdujo un nuevo término para definir lo mismo que ya se hacía. Pero quizá el factor que afectó más a la aceptación del concepto competencia, fue la importancia que le dieron las organizaciones empresariales, lo cual dio lugar a escepticismos y rechazo en distintos actores educativos e impidió apreciar sus ventajas didácticas.

10 Hay una tipología de competencias que hace una distinción entre competencias básicas o genéricas, que regularmente son entendidas como transversales, y competencias especificas. Trujillo (2014, p. 316) explica que las competencias específicas son aquellas que están directamente relacionadas con el ejercicio de ocupaciones concretas y no son fácilmente transferibles de uno a otro ámbito, dadas sus características.

11 Se han planteado diversas propuestas de guías metodológicas para la redacción de competencias. En este caso, sigo en buena medida la propuesta de Tobón (2012). 
de evaluación, identifiqué las distintas competencias que se espera que desarrollen los estudiantes en cada uno de sus momentos. En la tabla 1 presento las competencias argumentativas específicas identificadas, que reflejan la integración de los temas con sus estrategias didácticas.

Tabla 1. Competencias que se espera que los estudiantes desarrollen con el curso Lógica y Argumentación

\begin{tabular}{|c|c|c|}
\hline \multicolumn{3}{|c|}{ Competencias del primer momento del curso } \\
\hline Verbo & Objeto & Situación \\
\hline Distingue & $\begin{array}{l}\text { Las diferencias entre argumentar, } \\
\text { inferir y explicar, }\end{array}$ & $\begin{array}{l}\text { para identificarlas y discriminar su uso } \\
\text { en fragmentos de textos diversos. }\end{array}$ \\
\hline Identifica & $\begin{array}{l}\text { las características de la acción de } \\
\text { argumentar, }\end{array}$ & $\begin{array}{l}\text { para analizar situaciones en las } \\
\text { que podría haber o no un acto } \\
\text { argumentativo. }\end{array}$ \\
\hline Distingue & $\begin{array}{l}\text { las características de las orientaciones } \\
\text { dialéctica, lógica y lingüística en } \\
\text { argumentación }\end{array}$ & $\begin{array}{l}\text { para reconocer cuándo se adopta } \\
\text { alguna de ellas en algunas frases. }\end{array}$ \\
\hline Reconoce & $\begin{array}{l}\text { las características de un contexto de } \\
\text { explicación y otro de justificación, }\end{array}$ & $\begin{array}{l}\text { para describir cada uno de ambos } \\
\text { tipos de contexto respecto a una } \\
\text { misma frase. }\end{array}$ \\
\hline \multicolumn{3}{|c|}{ Competencias del segundo momento } \\
\hline Verbo & Objeto & Situación \\
\hline Define & $\begin{array}{l}\text { las premisas y la conclusión de un } \\
\text { argumento, }\end{array}$ & $\begin{array}{l}\text { para ser capaz de reconocerlas en el } \\
\text { análisis de párrafos argumentativos. }\end{array}$ \\
\hline Distingue & $\begin{array}{l}\text { la diferencia entre argumentos de tipo } \\
\text { fáctico, práctico o valorativo, }\end{array}$ & $\begin{array}{l}\text { para ser capaz de reconocerlos, } \\
\text { reconstruirlos y justificarlos a partir } \\
\text { del análisis de fragmentos. }\end{array}$ \\
\hline Reconoce & $\begin{array}{l}\text { las diferentes estructuras } \\
\text { argumentativas a las que puede } \\
\text { corresponder un argumento } \\
\text { (encadenamiento, coorientación y } \\
\text { metaargumentación) }\end{array}$ & $\begin{array}{l}\text { para elaborar un diagrama de la } \\
\text { estructura argumentativa presente, } \\
\text { resaltando sus elementos textuales, } \\
\text { al analizar un fragmento de texto } \\
\text { periodístico. }\end{array}$ \\
\hline \multicolumn{3}{|c|}{ Competencias particulares del tercer momento del curso } \\
\hline Verbo & Objeto & Situación \\
\hline Reconoce & $\begin{array}{l}\text { las características de las garantías, } \\
\text { explícitas o implícitas, de los } \\
\text { argumentos, }\end{array}$ & $\begin{array}{l}\text { para ser capaz de identificarlas } \\
\text { en los argumentos detectados en } \\
\text { fragmentos de texto. }\end{array}$ \\
\hline Identifica & $\begin{array}{l}\text { argumentos opuestos y el peso que } \\
\text { cada uno aporta a su tesis, }\end{array}$ & $\begin{array}{l}\text { para ser capaz de justificar cuál es } \\
\text { el argumento al que puede atribuir } \\
\text { mayor peso a través del análisis de } \\
\text { textos breves. }\end{array}$ \\
\hline
\end{tabular}


Continuación Tabla 1. Competencias que se espera que los estudiantes desarrollen con el curso Lógica y Argumentación

\begin{tabular}{c|l|l|}
\hline \multicolumn{2}{|c|}{ Competencias particulares del tercer momento del curso } \\
\hline Rerbo & \multicolumn{1}{|c|}{ Objeto } & \multicolumn{1}{c|}{ Situación } \\
\hline Reconoce & $\begin{array}{l}\text { la diferencia entre objetar, recusar, } \\
\text { refutar y contraargumentar, así } \\
\text { como las características de la } \\
\text { metaargumentación, y si las críticas } \\
\text { son lógicas, dialécticas o retóricas, }\end{array}$ & $\begin{array}{l}\text { para que pueda identificarlas en } \\
\text { fragmentos de textos específicos. }\end{array}$ \\
\hline Reconoce & $\begin{array}{l}\text { los criterios de identificación y } \\
\text { evaluación de argumentos }\end{array}$ & $\begin{array}{l}\text { para ser capaz de buscar por su } \\
\text { cuenta un argumento o construirlo, } \\
\text { a fin de ilustrar esquemas } \\
\text { argumentativos distintos y mostrar sus } \\
\text { elementos. }\end{array}$ \\
\hline
\end{tabular}

Al término de cada momento del desarrollo del curso, cada estudiante realiza un test, que es el principal instrumento de evaluación del curso. ${ }^{12}$ Los estudiantes deben entregar sus respuestas una vez concluida la revisión de los temas correspondientes a cada uno de los momentos del curso. Cabe resaltar que los estudiantes conocen las preguntas de cada test desde el inicio de la revisión de los temas, de tal manera que pueden decidir si van resolviendo cada ítem en el momento en que se aborda el tema correspondiente en clase o si los resuelven todos al concluir la revisión de los temas del periodo a evaluar.

En la tabla 2 presento el contenido general de los ítems que integran cada uno de los test.

\begin{tabular}{|c|c|c|c|}
\hline & Test 1 & Test 2 & Test 3 \\
\hline Ítem 1 & $\begin{array}{l}\text { Diferenciar (en } \\
\text { cuatro fragmentos } \\
\text { de textos) si se } \\
\text { argumenta, razona, } \\
\text { infiere, explica o se } \\
\text { dan razones. }\end{array}$ & $\begin{array}{l}\text { Identificar las premisas } \\
\text { y la conclusión de } \\
\text { los argumentos } \\
\text { contenidos en cuatro } \\
\text { párrafos que se } \\
\text { proponen. }\end{array}$ & $\begin{array}{l}\text { Identificar al argumento } \\
\text { que se critica y el tipo } \\
\text { de crítica que se le } \\
\text { esté haciendo. En la } \\
\text { identificación de los } \\
\text { argumentos de un grupo } \\
\text { de cuatro fragmentos. }\end{array}$ \\
\hline Ítem 2 & $\begin{array}{l}\text { Seleccionar (de } \\
\text { un grupo de cinco } \\
\text { opciones) la que le } \\
\text { parece apropiada } \\
\text { para definir lo que es } \\
\text { argumentar y ofrecer } \\
\text { su justificación. }\end{array}$ & $\begin{array}{l}\text { Distinguir si se trata de } \\
\text { argumentos fácticos, } \\
\text { prácticos o valorativos, } \\
\text { según corresponde } \\
\text { en un grupo de } \\
\text { cuatro argumentos } \\
\text { propuestos. }\end{array}$ & $\begin{array}{l}\text { Hacer explícitas las } \\
\text { garantías de los } \\
\text { argumentos de textos } \\
\text { o proponer una. En la } \\
\text { identificación de los } \\
\text { argumentos de un grupo } \\
\text { de cuatro fragmentos. }\end{array}$ \\
\hline
\end{tabular}

12 Para complementar la evaluación se tienen en cuenta la asistencia y las participaciones de los estudiantes en clase. 


\begin{tabular}{|c|c|c|c|}
\hline & Test 1 & Test 2 & Test 3 \\
\hline Ítem 3 & $\begin{array}{l}\text { - Describir un } \\
\text { contexto que } \\
\text { exprese una } \\
\text { explicación y otro } \\
\text { en el que exprese } \\
\text { una justificación } \\
\text { de la frase que se } \\
\text { le propone. }\end{array}$ & $\begin{array}{l}\text { Identificar las } \\
\text { estructuras } \\
\text { argumentativas, los } \\
\text { elementos textuales } \\
\text { que permiten } \\
\text { reconocer esas } \\
\text { relaciones, para } \\
\text { después construir el } \\
\text { diagrama de cada } \\
\text { argumentación, } \\
\text { en un grupo de } \\
\text { cinco fragmentos } \\
\text { propuestos. }\end{array}$ & $\begin{array}{l}\text { Identificar las premisas, las } \\
\text { conclusiones, las garantías } \\
\text { de los argumentos } \\
\text { opuestos de un texto } \\
\text { específico que se propone y } \\
\text { se pide que se indique por } \\
\text { qué se atribuye más peso a } \\
\text { uno que a otro y cómo se } \\
\text { justifica esa preferencia. }\end{array}$ \\
\hline Ítem 4 & $\begin{array}{l}\text { Reconocer la } \\
\text { perspectiva sobre la } \\
\text { argumentación que } \\
\text { se adopta (ya sea: } \\
\text { dialéctica, lógica, } \\
\text { retórica y lingüística) } \\
\text { en cada una de los } \\
\text { cuatro fragmentos } \\
\text { que se ofrecen. }\end{array}$ & & $\begin{array}{l}\text { Construir o buscar } \\
\text { dos argumentos que } \\
\text { ilustren dos esquemas } \\
\text { argumentativos } \\
\text { distintos, en los que } \\
\text { deben indicar cuál es } \\
\text { el esquema ilustrado } \\
\text { por cada uno, hacer el } \\
\text { diagrama correspondiente, } \\
\text { mostrando sus premisas, su } \\
\text { conclusión y su garantía. } \\
\text { Formular una posible } \\
\text { recusación de uno de ellos } \\
\text { y una posible refutación del } \\
\text { otro, cuidando que sean } \\
\text { apropiadas y no triviales. }\end{array}$ \\
\hline Ponderación & 2 puntos & 3 puntos & 4 puntos \\
\hline
\end{tabular}

Aunque la revisión específica de las competencias a desarrollar en cada momento del curso permite identificar la estrategia didáctica con la que se aborda cada contenido temático, ello no agota las consideraciones didácticas. Cabe resaltar también otros aspectos didácticos, como, por ejemplo, que los estudiantes cuentan con un compendio de los contenidos teóricos del curso y con las presentaciones que utiliza el profesor en sus exposiciones, disponibles en la plataforma virtual de la Universidad. Asimismo, que los ejemplos y ejercicios planteados en clase son muy cercanos a los que contienen los test, y al abordar cada tema se ofrecen distintos ejemplos y ejercicios a partir del análisis de fragmentos de textos diversos. Aunque la mayoría de los estudiantes que toman la clase son del grado en filosofía, no se exploran textos exclusivamente de filosofía, sino que la mayor parte de los materiales provienen de artículos periodísticos o de notas de revistas sobre temas variados.

Es importante advertir que muchas de las decisiones didácticas del curso se tomaron considerando las necesidades de la institución y las 
características de los alumnos, atendiendo a su diversidad, así como a los recursos disponibles. De tal forma que, como se ha podido ver, existe una coherencia interesante entre lo planteado en el objetivo central del curso, las competencias a desarrollar en cada uno de sus momentos, los contenidos de los ítems de cada uno de los test con los que se mide el progreso de los estudiantes y la integración del modelo dialéctico argumental.

\section{Revisión y reflexiones sobre algunos resultados}

Aunque una revisión cuantitativa, como se observará más adelante, no es suficiente para evaluar los resultados que están mostrando los alumnos al curso Lógica y Argumentación, al menos puede ofrecer algunos indicadores y elementos de reflexión. A continuación, presentaré mi análisis de los valores arrojados en la resolución de los test realizados por los estudiantes de los dos grupos -110 y 160 , matutino y vespertino respectivamente- durante tres años (de 2017 a 2019). De modo que la muestra total revisada corresponde a un universo de 205 alumnos. ${ }^{13}$

El análisis de los resultados generales por periodo y año arrojó la información que se aprecia en la figura 1.

Figura 1. Concentrado de los promedios obtenidos por periodo por los dos grupos

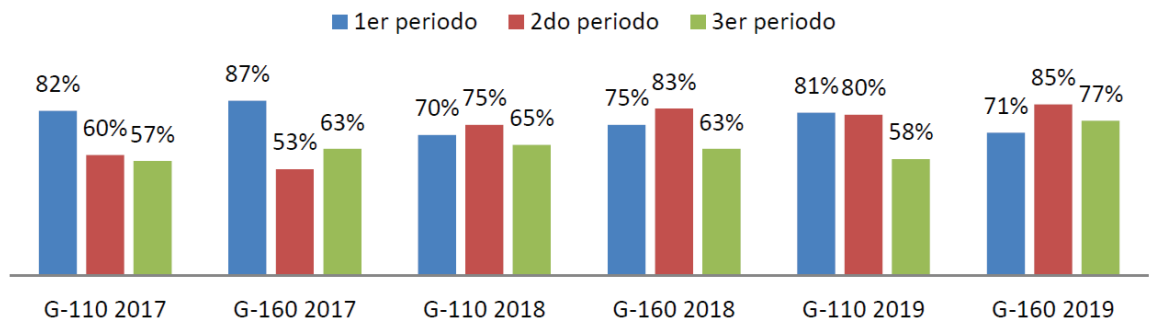

En el entendido de que los resultados de los test refieren al nivel de desarrollo de las competencias que aspiraban a medir, entre los aspectos que nos permite apreciar la gráfica, vemos que hay una buena respuesta en cuanto al desarrollo de las competencias argumentativas más básicas, que fue medido por el primer test, pues en ningún caso los grupos obtuvieron un promedio inferior al $71 \%$. En el paso de las competencias del primer periodo al segundo, vemos que en algunos grupos hubo incluso un mejor desempeño que en el primer momento (en el grupo 160, en los cursos de 2018 y 2019, se alcanzaron promedios de 83 y $85 \%$, respectivamente), pero en otros grupos el desempeño fue inferior al conseguido en el primer

13 Analicé los datos arrojados por los test a través de las medidas de tendencia central y de dispersión o variabilidad. Dado que entre los test había diferencias de ponderación, por la variación de la dificultad de los ítems, para tener una base común, todos los resultados se transformaron a valores en decimales. 
periodo (el grupo 110 en el año 2017 obtuvo un promedio del $60 \%$ y en ese mismo año el grupo 160 obtuvo un $53 \%$ ). Respecto al desarrollo de las competencias del tercer periodo, vemos que fue el periodo que ofreció la mayor dificultad, pues presentó los menores porcentajes de desempeño en la mayoría de los casos (con porcentajes del 57 al $65 \%$ en la mayor parte de la muestra).

La gráfica también nos permite ver que no hay regularidades absolutas en el desempeño de los grupos en los distintos periodos del curso durante los años examinados. Por ejemplo, el grupo 160 en el año 2017 tuvo su desempeño más bajo en el segundo periodo, con un $53 \%$; mientras que el mismo grupo, pero en el año 2019, en el mismo segundo periodo, obtuvo su mejor desempeño del curso, logrando un promedio del $85 \%$. Esto indica que se requiere información adicional para comprender las causas del comportamiento variable de cada grupo en los distintos ańos, o para comprender el desempeño de un mismo grupo a lo largo de los tres momentos del curso. Se requiere más información para comprender, por ejemplo, por qué el grupo 160, en el año 2019, obtuvo en el primer periodo un rendimiento del $71 \%$ (uno de los dos promedios más bajos de toda la muestra durante el primer periodo), mientras que en el tercer periodo obtuvo un promedio del $77 \%$ (el promedio más alto de toda la muestra durante el tercer periodo, que como señalé anteriormente, es el de mayor complejidad).

El análisis de los promedios globales del curso, por grupo, arrojó los resultados que se muestran en la figura 2 .

Figura 2. Concentrado de los promedios globales obtenidos por los dos grupos



El promedio global debería ser un indicador del desarrollo de una posible competencia global, que -dada la revisión que he hecho de los contenidos y competencias de cada momento del curso- sería una competencia encaminada a que el alumno: «distinga las características de la acción de argumentar (sus tipos y enfoques de estudio), los elementos de análisis de argumentaciones complejas (garantías, esquemas argumentativos, metaargumentación) así como los criterios de su evaluación (contraargumentaciones y recursos de ponderación de argumentos), para ser capaz de identificar, reconstruir, criticar y justificar su valoración sobre los argumentos que localiza en fragmentos de textos diversos o respecto de aquellos que es capaz de construir.» 
No es claro hasta qué punto podemos afirmar que el promedio de los valores obtenidos en las tres pruebas refleje efectivamente el desarrollo de una competencia global, en los términos en los que la he enunciado. En cualquier caso, se trata solo de un indicador que nos lleva a considerar que el desarrollo general de las competencias argumentativas que propone el curso en ningún caso fue inferior al $63 \%$, y que el mayor promedio global fue del $77 \%$, logrado por el grupo 160 en el año 2019. Si contrastamos estos promedios globales con los promedios obtenidos durante el tercer periodo -que observamos como el periodo de mayor complejidad- los cuales oscilaron entre el 57 y el $77 \%$, vemos que entre los menores porcentajes alcanzados solo hay una diferencia del $6 \%$ y que los porcentajes más altos coinciden con el $77 \%$.

Otro dato importante a observar es que el promedio global de los promedios del curso, respecto a toda la muestra, es de $67 \%$ y el rango global nos arroja una variación de 4,3, con un promedio de las modas globales de 7,3 . La poca variación entre los datos, con una moda del promedio global del 7,3 y con un porcentaje global total del $67 \%$ ofrece un panorama alentador del nivel de comprensión y el desarrollo de competencias que han mostrado los estudiantes que han cursado la asignatura.

Más allá de los datos estadísticos de la medición de los test, vale la pena mencionar las cifras relacionadas con la aprobación del curso, pues la aprobación tiene un porcentaje amplio; únicamente en uno de los años considerados y solo en uno de los grupos, la aprobación fue del $74 \%$, mientras que en el resto de los casos la aprobación llegó a ser incluso del $94 \%$.

Es posible reforzar la revisión cuantitativa de los resultados de los test examinados, ${ }^{14}$ pero ni siquiera en ese caso podría ofrecernos la información que permita conocer con detalle las razones que explican la diferencia entre el menor y el mayor desempeño grupal, y menos aún aquella que nos permita identificar las razones que expliquen los mejores desempeños individuales entre los estudiantes. Para obtener una información más amplia, se requeriría, por lo menos, la aplicación de instrumentos de investigación cualitativa. El ideal sería desarrollar investigación educativa que explicara la relación entre las competencias argumentativas con las que ya contaban los alumnos al inicio del curso y las que hayan desarrollado al término del mismo, de tal manera que permita contrastarlas, e incluso aplicar algún instrumento de recogida de información uno o dos años después de haberse cursado la asignatura.

\section{Investigación y enseñanza en el trabajo de Hubert Marraud}

No es el propósito de este apartado hacer una revisión pormenorizada del trabajo de investigación que Hubert Marraud ha aportado a la teoría de la argumentación; aunque esa sea una tarea necesaria, va más allá del propósito

14 Como al identificar los puntajes obtenidos en cada ítem de cada uno de los test, que nos daría información específica sobre el desarrollo de cada una de las competencias definidas. 
de este artículo. Tan solo espero destacar algunos elementos de la producción de Marraud que ayuden a evidenciar que el diseño de su actual curso de argumentación se ha beneficiado de sus procesos de investigación, de igual forma que su producción teórica se ha visto favorecida por el ejercicio docente que ha venido desarrollando en su curso universitario de argumentación. Tomando en cuenta el beneficio recíproco que podemos observar en el caso examinado, vale la pena reflexionar un poco sobre si es posible sugerir alguna razón especial de ese mutuo beneficio y si en ello tiene algo que ver que se presente en el campo de la teoría de la argumentación.

Durante 2004, Hubert Marraud tuvo oportunidad de dictar una asignatura optativa en la UAM, en la que ofreció herramientas para el análisis de la argumentación filosófica, y de los materiales que preparó para ese curso surgió su Methodus Argumetandi, manual práctico para el estudio del discurso argumentativo, que sería publicado por ediciones de la UAM en 2007. Un poco antes, en 2006, publicó «Lógica y argumentación. La estructura de la argumentación», que es, dentro de su obra publicada, el primer artículo en el que se aparta de la investigación en lógica formal que venía desarrollando y se orienta a lo que él mismo anunciaba como un trabajo de lógica informal. ${ }^{15}$ En 2007 publicó el artículo «La analogía como transferencia argumentativa»y, como señalé antes, en ese mismo año se dio a conocer su Metodus argumentandi, obra en la que, si bien ya ofrecía criterios para determinar la calidad de los argumentos que surgían de la práctica argumentativa cotidiana, aún tomaba en cuenta algunas consideraciones formales para la evaluación de las inferencias.

En 2010 Marraud comenzó a impartir su curso Lógica y Argumentación en la licenciatura en filosofía en la UAM y así tuvo la ocasión ideal para explicar y examinar las herramientas que proponía para el análisis y evaluación de argumentos; a la par, continuó su producción de artículos de investigación, como «Argumentos suposicionales, razones y premisas» (2010), «Las razones del necio» (2012) y «Variedades de la argumentación a fortori» (2013). De los materiales que preparó para su nuevo curso surgió su iEslogic@?: análisis y evaluación de argumentos (2013), manual en el que proporciona un aparato conceptual más sólido para el análisis y evaluación de argumentos, y en el que es posible detectar la configuración de las tres fases que plantea en su curso universitario: la primera, para la identificación de argumentos; la segunda, para comprender su estructura, y la tercera, para evaluarlos. A la vez que dictaba su curso, maduraba su propuesta de dialéctica argumental, que desarrolló en su artículo «Do Arguers Dream of Logical Standards? Arguers' dialectic vs. Arguments' dialectic» (2015).

Marraud ha continuado con su producción de artículos de investigación sobre diversos tópicos del amplio campo de la teoría de la argumentación y, de forma paralela, la propuesta didáctica ofrecida en ¿Es lógic@? fue objeto de revisión y refinamientos en su Breve curso de teoría de los argumentos (2014).

15 Hubert Marraud hace referencia explícita de su trabajo de investigación como parte del campo de la teoría de la argumentación hasta sus artículos de 2012 y 2013. 
En otros trabajos revisa con mayor detenimiento herramientas específicas, como en el artículo «Diagramación de argumentos» (2016) y en su Guía de campo de los esquemas argumentativos (2017a). Toda esa producción ha estado acompañada del desarrollo de su curso universitario de argumentación y, con ello, de la necesidad de seguir revisando y mejorando su propuesta didáctica, como se puede apreciar en todas las modificaciones concretadas en su compendio Conceptos y técnicas para el análisis de argumentos (2019), texto que ocupó centralmente en su práctica docente de ese mismo año. ${ }^{16}$

He mencionado solo algunos de los textos de Hubert Marraud, que ayudan a observar el beneficio de combinar investigación y enseñanza. Resalto que tal beneficio es mutuo, porque el trabajo de investigación desarrollado por Marraud sobre distintos tópicos en teoría de la argumentación, luego se ha reflejado en las modificaciones de sus manuales, que han formado parte indispensable del material empleado en sus cursos. Al mismo tiempo, las exigencias propias de mantener actualizado su curso, considerando las características de sus alumnos, se pueden advertir en el tipo de ejemplos, explicaciones y observaciones, que en algunas ocasiones surgen de las reflexiones conjuntas con sus estudiantes o del análisis de los ejercicios abordados en clase.

Es importante resaltar que el curso de argumentación de Marraud no es la mera exposición de su modelo de dialéctica argumental, porque en el desarrollo del curso ofrece respuesta a planteamientos teóricos, al mismo tiempo que, como profesor, resuelve problemáticas relativas a la transferencia de esos conceptos durante las prácticas en el aula. Y entre más cercanas sean esas prácticas a los procesos argumentativos que realizan los estudiantes en su cotidianidad, mejor es la transferencia de conocimientos. En cuanto a esa transferencia, cabe destacar, además, que la evaluación de argumentos que propone el curso no parte de criterios planteados como reglas preestablecidas e ideales, sino que más bien se plantean criterios de ponderación orientados al examen de argumentos que se dan en la práctica de intercambios argumentativos. ${ }^{17} \mathrm{De}$ ahí que resulte especialmente valioso el ejercicio docente y el intercambio colaborativo con los estudiantes, aunque no sea fácil apreciar cuánto ha sido de ayuda para el refinamiento que Hubert Marraud ha ido plasmando en la producción de sus manuales.

La revisión de la investigación y enseñanza de Marraud ha hecho que me pregunte si el modo singular en el que en su caso se ha presentado un muto beneficio entre ambos factores tiene alguna razón especial. Aunque es claro que la revisión de un caso, como el que hemos examinado aquí, no puede ofrecer información contundente, creo que al menos aporta elementos para sugerir que los lazos entre investigación y enseñanza son

16 El trabajo de revisión y profundización de la propuesta teórica y didáctica de la dialéctica argumental de Marraud continúa; de hecho, pronto se difundirá un nuevo manual de su modelo, que se encuentra en prensa.

17 Agradezco al revisor anónimo de Polisemia hacerme notar la pertinencia de destacar los rasgos de la enseñanza y del tipo de propuesta de evaluación del curso de Hubert Marraud. 
más fuertes y fructíferos si el interés de tal enseñanza se orienta hacia la aplicación de los conocimientos, no solo en un contexto académico, sino precisamente, y sobre todo, más allá de él. Pero, además del componente práctico, considero importante observar que, según parece, la investigación en el campo de la teoría de la argumentación, centrada en el ejercicio de las prácticas argumentativas - tal cual se presentan, incluso en un aula universitaria- es un hecho que fortalece de modo singular el vínculo que pueda darse entre investigación y enseñanza.

Ello es así, por cuanto el contexto educativo universitario en el que se lleva a cabo un curso de argumentación no deja de formar parte de un espacio de prácticas argumentativas, en el que las herramientas que ofrece el curso cobran su sentido práctico y al mismo tiempo proporcionan al docente-investigador información para continuar su trabajo de revisión, análisis y reformulación teórica. Esto es, el propio investigador de las prácticas argumentativas es también actor de dichas prácticas, así que su propia práctica argumentativa puede formar parte de su objeto de estudio. De modo que al dictar un curso de argumentación, que no se limita a un enfoque puramente teórico, sino que además proporciona instrumentos para mejorar la práctica argumentativa, durante ese ejercicio docente, el profesor-investigador recibe información y retroalimentación de la práctica argumentativa de sus estudiantes, de su propia práctica argumentativa y de la interacción argumentativa con ellos. Ese aspecto singular hace que la retroalimentación que recibe el docente-investigador en el proceso educativo sea una valiosa contribución para progresar en sus investigaciones y que luego, tales investigaciones, sirvan para mejorar la enseñanza. ${ }^{18}$

\section{Conclusiones}

Un curso universitario de argumentación, como el que desarrolla la propuesta de la dialéctica argumental de Hubert Marraud, esclarece el carácter dialéctico de los argumentos, mostrando que entre ellos hay relaciones de oposición, reforzamiento o crítica, y que la suma de argumentos significa siempre un incremento en la complejidad del análisis necesario para evaluarlos, ya

18 Me interesa destacar que esa singular interacción entre investigación y enseñanza, entre teoría y práctica, que se presenta en el campo de la teoría de la argumentación, puede ser un elemento importante para clarificar la diferencia -que durante largo tiempo ha sido confusa- en el interés que se tiene por los argumentos tanto en el campo de la teoría de la argumentación como en el de la lógica formal clásica; e incluso, en trabajos de lógica formal no clásica pero que mantienen un interés exclusivo en consideraciones estructurales de las inferencias, sin tener en cuenta otros elementos que forman parte de las prácticas argumentativas. La confusión proviene, supongo, del hecho de que en distintos manuales de lógica formal se señala que su objeto de estudio son los "argumentos", como los que podríamos encontrar en cualquier "proceso argumentativo", cuando en realidad están hablando de "argumentos" en un sentido muy particular, ya que su interés se centra en modelar únicamente los procesos inferenciales o de razonamiento y no en recuperar las "prácticas argumentativas" tomando en cuenta las consideraciones retóricas y dialécticas que les son características. Es importante aumentar los esfuerzos de investigación para avanzar en la clarificación indicada. Mi única intención por el momento, es hacerlo notar. 
que es preciso distinguir si entre ellos hay, por ejemplo, encadenamiento, coorientación o metaargumentación. El análisis evaluativo de los argumentos supone valorar su fuerza inferencial y, si es posible, realizar una comparación adecuada entre ellos, dependiendo del tipo que presentan y la identificación de las finalidades que persiguen.

Como hemos visto, el curso Lógica y Argumentación es un curso bien estructurado, que mantiene una coherencia interesante entre su objetivo central, sus materiales y sus mecanismos de evaluación. Los elementos cuantitativos que ofrezco en este artículo para tener alguna referencia sobre la respuesta que están teniendo los estudiantes ante el curso, son insuficientes, pero sí muestran algunos indicios de que la respuesta al curso es favorable. Lo ideal sería contar con una investigación educativa de más amplio espectro, empleando herramientas que no solo sean cuantitativas, para profundizar en el conocimiento de los resultados logrados en el curso.

El examen de algunos aspectos del trabajo de investigación y enseñanza que ha venido realizando Hubert Marraud, permite poner de relieve que en el campo de la teoría de la argumentación, al parecer de un modo singular, puede darse un beneficio mutuo al combinar investigación y enseñanza, de tal manera que ambas áreas de trabajo se vean fortalecidas. Considero que el análisis de este caso se convierte, al menos, en una invitación para investigadores y docentes, a prestar atención y abrirse a la posibilidad de experimentar ese muto beneficio.

La revisión hecha al curso Lógica y Argumentación muestra que la enseñanza universitaria de la argumentación pone retos a la investigación y a la teorización en el campo de la teoría de la argumentación, pues le demanda herramientas específicas para el análisis y evaluación de argumentos. Pero, al mismo tiempo, los esfuerzos de investigación en este campo fortalecen su didáctica, puesto que proporcionan las bases para crear nuevos materiales y modelos de enseñanza.

\section{Agradecimientos}

Este trabajo fue posible gracias al apoyo del programa PASPA de la DGAPA de la UNAM para realizar una estancia de investigación posdoctoral en la UAM. Agradezco igualmente el apoyo académico del Proyecto de Investigación DGAPA IN401619 «Lógicas no clásicas y argumentación en ciencias», en el cual participo.

\section{Referencias}

Bermejo-Luque, L. (2018). El modelo normativo-lingüístico de argumentación. Cogency, 9(2), 7-30.

Manso, J., y Sánchez-Tarazaga, L. (2020). El aprendizaje por competencias en las aulas de infantil y primaria [Capitulo de libro]. En prensa. 
Marraud, H. (2006). Lógica y argumentación. La estructura de la argumentación. Azafea. Revista de Filosofía, (8), 103-120. https://doi.org/10.14201/628

Marraud, H. (2007). Metodus argumentandi. Universidad Autónoma de Madrid. http://dx.doi.org/10.15366/m.argumentandi2014

Marraud, H. (2010). Argumentos suposicionales, razones y premisas. Tópicos, (39), 153-165. https://doi.org/10.21555/top.v39i1.102

Marraud, H. (2012). Las razones del necio. Bajo Palabra. Revista de Filosofía, Epoca $2(7), 533-554$.

Marraud, H. (2013a).¿Es lógic@? Análisis y evaluación de argumentos. Cátedra.

Marraud, H. (2013b). Variedades de la argumentación a fortiori. Revista Iberoamericana de Argumentación, (6), 2013, 127-144.

Marraud, H. (2014, julio). Breve curso de teoria de los argumentos. https://www. academia.edu/8189164

Marraud, H. (2015). Do arguers dream of logical standars? Arguers' dialectic vs. arguments' dialectic. Revista Iberoamericana de Argumentación, (10),1-18.

Marraud, H. (2016). Diagramación de argumentos. Revista Iberoamericana de Argumentación. (12), 1-25.

Marraud, H. (2017a). Guia de campo de esquemas argumentativos. https://www. academia.edu/31254695

Marraud, H. (2017b). Una visión dialéctica de los contraargumentos. https:// www.academia.edu/35125623

Marraud, H. (2019). Conceptos y técnicas para el análisis de argumentos. https://www. academia.edu/37244768

Sánchez-Tarazaga, L. (2017). Las competencias docentes del profesorado de educación secundaria importancia percibida e implicaciones en la formación inicial [Tesis doctoral, Universidad Jaume I]. https://www.tdx.cat/handle/10803/404324

Tobón, F. (2012). Manual con verbos para la redacción de competencias, criterios $y$ evidencias. CIFE, Centro Universitario. https://issuu.com/cife/docs/ manual_basico_de_redaccion_de_compe

Trujillo, J. (2014). El enfoque por competencias y la mejora de la educación, $R a$ Ximbai, 10(5), 307-322.

Vega Reñón, L. (2017). Lógica para ciudadanos. Ensayos de Lógica Civil. Madrid: Editorial Académica Española.

Wenzel, J. (2006). Three perspectives on argument. En R. Trapp y J. Schuetz (Eds.), Perspectives on argumentation: essays in honor of Wayne Brockriede (pp. 9-26). (Original publicado en 1990).

Wenzel, J. (1992). Perspectives on argument. En W. L. Benoit y D. Hample (Eds.), Reading in argumentation (pp. 121-144). Gruyter. 